\title{
Les empreintes génétiques : la révolution permanente
}

Les empreintes génétiques ont connu un développement prodigieux depuis leur description par Jeffreys et al. en $1985\left(\mathrm{~m} / \mathrm{s} n^{\circ} 6\right.$, vol. 1, p. 333). Ce développement éclate en directions multiples qu'on peut schématiser en deux voies principales, qui d'ailleurs s'entrecroisent souvent : la plus popularisée concerne les applications pratiques. En biologie humaine, elle permet d'identifier les relations familiales ou de suivre les suites d'une greffe de moelle osseuse. On a même abusé de la méthode en médecine légale, et des procès retentissants ont mis en garde, non pas contre le principe, mais contre des applications insuffisamment entourées de garanties techniques $\left(\mathrm{m} / \mathrm{s} n^{\circ} 8\right.$, vol. 5, p. 593). L'utilisation déborde largement l'espèce humaine et s'attaque aussi bien aux vertébrés de toutes espèces qu'aux invertébrés et aux végétaux. On a pu, par exemple, élucider les mœurs reproductrices de mammifères marins, phoques ou baleines, souvent rebelles à l'observation directe. La deuxième voie poursuit un objectif plus fondamental : par quels mécanismes se créent les différences de taille qui sont à la base des empreintes, et peut-on évaluer la vitesse des mutations qui les engendrent?

Au départ, la technique des empreintes génétiques s'appuie sur l'existence - dans le génome - de zones hypervariables en taille, la variabilité provenant du nombre de répétitions, en tandem, de courtes séquences. Jeffreys et al. les ont appelées minisatellites, en raison de leur analogie de structure avec l'ADN satellite antérieurement connu. D'autres les nomment VNTR (variable numbers of tandem repeats). On peut les déceler en fragmentant l'ADN par des enzymes de restriction qui ne coupent pas à l'intérieur des minisatellites. Dans le travail qui a lancé la méthode, les ceptible de reconnaître un grand nombre de minisatellites dispersés dans le génome. Mais on a aussi identifié certains loci doués d'une variabilité telle qu'ils peuvent à eux seuls servir de base à un système d'identification.

C'est ainsi que, par exemple, les VNTR peuvent remplacer le Southern blot classique pour l'étude du gène de l'apolipoprotéine B [1] ou de l'amylase [2].

- L'échelle. Dès le début de leur emploi, les empreintes ne réclamaient qu'une dose faible d'ADN, de l'ordre du microgramme. Il est aujourd'hui possible d'envisager une analyse de l'ADN à partir d'une seule cellule. Grâce à la méthode de la PCR, on peut amplifier avec fidélité un bloc entier d'unités répétitives, allant jusqu'à $5 \mathrm{~kb}$ et davantage, bien que le rendement diminue à mesure que la séquence à recopier s'allonge.

- Les mutations dans les minisatellites. Des mutations dans les lignées germinale et somatique ont été observées chez l'homme et la souris. Elles se traduisent par un changement de taille d'une des bandes, par addition ou soustraction d'une ou plusieurs des unités répétitives. Des mutations de la lignée germinale ont été suivies pour cinq loci différents par étude de familles collectées au CEPH (Centre d'étude du polymorphisme humain, Paris), devenu indispensable pour ce type de travail [3]. Une de ces mutations a été analysée avec précision, elle est due à la perte d'une seule unité [4]. L'intérêt de ce travail est qu'il battait en brèche, pour la première fois, l'hypothèse la plus communément admise pour expliquer ces mutations, celle d'un crossing over inégal à la méiose. Cette hypothèse implique qu'un échange de séquences flanquantes devrait avoir lieu entre deux chromosomes homologues, ce qui n'a pas été observé $(\mathrm{m} / \mathrm{s}$, Lexi- que, 1990, p. 14). Afin de ne pas conclure sur un seul exemple, une collaboration entre les équipes de Jeffreys et de R. White [5] utilisa le marqueur D1S7. Ce locus situé sur le chromosome 1 montre le taux de mutation le plus élevé de tous les VNTR connus, avec $5 \%$ des gamètes porteurs de nouveaux allèles. Trente-six allèles nouveaux avaient été observés [3]. Les parents de 12 d'entre eux étaient informatifs pour les marqueurs flanquants; chez 11 sur 12, aucune indication de recombinaison ne fut obtenue, et, dans chacun de ces cas, on a pu assigner avec précision le gain ou la perte d'unités au père ou à la mère.

A côté des mutations de la lignée germinale, des mutations somatiques ont été également observées [6]. Dans des lignées lymphoblastoïdes, les mutations se sont avérées très rares. En revanche, dans des tumeurs gastro-intestinales, elles ont fait preuve d'une grande fréquence : 10 allèles mutants sur 39 tumeurs colorectales, 8 sur 11 tumeurs gastriques, contre un seul mutant sur 38 cancers du sein. Des mutations germinales et somatiques ont été également détectées en utilisant un locus instable de la souris [7].

La possibilité de mutations met-elle en cause la méthode des empreintes génétiques, pour les études de filiation, par exemple, puisqu'elle postule une transmission mendélienne du nombre de bandes? Il ne le semble pas car, dans les tissus normaux qui, contrairement aux cancers, sont polyclonaux - des allèles mutants seraient très hétérogènes en taille et ne seraient pas détectables en partant d'un tissu entier. Il faudra cependant se méfier lorsqu'on amplifiera un échantillon minirne d'ADN.

- Vers l'infiniment petit. Est-il possible de pénétrer davantage dans l'intimité des minisatellites, et 
d'abord d'en explorer l'hétérogénéité éventuelle ? Jeffreys et al. [8] ont étudié le clone MS32, qui détecte un locus hypervariable D1S8 situé sur le chromosome 1 en 1q42-q43 chez 80 Anglais et un échantillonnage du $\mathrm{CEPH}$. Il fallait trouver une méthode rapide pour détecter des polymorphismes autres que de taille. La séquence de l'unité de répétition, de $29 \mathrm{pb}$, montre une coupure dans chaque unité par l'enzyme HinfI, et dans un certain nombre, mais non dans toutes, une autre coupure par HaeIII due à une transition $\mathrm{A} \rightarrow \mathrm{G}$. On isole l'ensemble du minisatellite par coupure à l'aide de l'enzyme SauIIIA ; puis, après amplification et marquage, les allèles subissent une digestion progressive par chacune des deux enzymes : après contact pendant 10 à 60 secondes, des aliquotes sont prélevées, mélangées et soumises à électrophorèse. La digestion par HinfI fournit une échelle continue de fragments dont on déduit le nombre d'unités de répétition. La comparaison des deux pistes permet de savoir, pour chaque unité, si elle est ou non coupée par HaeIII. On peut représenter les résultats (figure 1), soit par une carte, soit par une notation arbitraire : les auteurs proposent d'appeler a les unités coupées par HaeIII, et $\mathrm{t}$ celles qui ne le sont pas. La méthode permet de cartographier jusqu'à 240 unités, et la notation proposée distingue de nombreux haplotypes variant par leur longueur et la distribution des a et des t. Par cette technique, on pourrait distinguer un nombre fantastique de combinaisons différentes, de l'ordre de $10^{70}$. C'est ce que Dover [9] appelle " empreintes à l'intérieur d'empreintes".

Mais il est encore possible d'aller plus loin pour tenter de comprendre le mécanisme de ces réarrangements. En séparant des molécules selon leur taille sur gel d'agarose, on a isolé des mutants de délétion que l'on a amplifiés et analysés. Presque toujours, ces molécules semblent avoir subi une délétion unique d'un certain nombre d'unités. Jamais on n'a obtenu d'indications de recombinaison entre deux allèles, méiotique ou mitotique.

Les conclusions qu'on peut tirer de cet ensemble de travaux sont nombreuses. D'abord, l'hétérogénéité biologique des minisatellites est encore plus grande qu'on ne le pensait et défie l'imagination. Ensuite, l'origine de cette hétérogénéité ne tient sans doute pas à des crossing over inégaux à la méiose, et on ne possède à son endroit que des hypothèses : échanges entre chromatides sœurs, conversions géniques, "glissements" de la polymérase. Comme ces divers mécanismes ne sont pas exclusifs les uns des autres, et que ces minisatellites, extraordinairement variables, ne sont que la partie émergée de multiples familles encore inconnues, bien des surprises attendent sans doute les candidats séquenceurs du génome [10]. Enfin, et ce n'est pas le moins

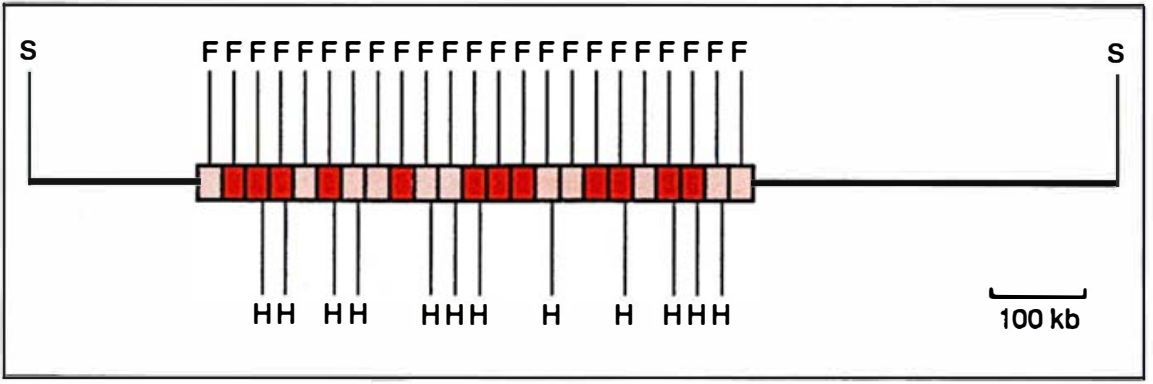

Figure 1. Analyse des unités répétitives dans un allèle du minisatellite MS32 détectant le locus D1S8. Carrés ouverts : unités de répétition; $S$ : coupures par SaulllA ; F : coupure par Hinfl (dans toutes les unités) ; H : coupure par Haelll; lecture en notation a et t:ttaataattaaattattataaat (modifié d'après [8]).

$\mathrm{m} / \mathrm{s} n^{\circ} 7$ vol. 6 , septembre 90 important, les allèles du locus D1S8 - et certainement de bien d'autres loci - émanent apparemment de lignées haploïdes, et devraient donc permettre de construire des cartes phylogéniques. Jusqu'à présent, on ne pouvait utiliser à cet effet que le génome mitochondrial, car l'évolution de l'ADN nucléaire est trop lente. Celle de l'ADN des minisatellites, beaucoup plus rapide, pourra être mise à profit dans un proche avenir pour les études de génétique des populations.

\section{J.-C. D.}

1. Boerwinkle E, Xiong W, Fourest E, Chan L. Rapid typing of tandemly repeated hypervariable loci by the polymerase chain reaction application to the apolipoprotein $\beta$ 3' hypervariable region. Proc Natl Acad Sci USA 1989 ; 86: 212-6.

2. Dracopoli NC, Meisler MH. Mapping the human amylase gene cluster on the proximal short arm of chromosome 1 using a highly informative (CA)n repeat. Genomics $1990 ; 7$. 97-102.

3. Jeffreys A, Royle N, Wilson V, Wong Z. Spontaneous mutation rates to new length alleles at tandem-repetitive hypervariable loci in human DNA. Nature 1988 ; 332 : 278-81. 4. Wolff RK, Nakamura Y, White R. Molecular characterization of a spontaneously generated new allele at a VNTR locus: no exchange of flanking DNA sequence. Genomics $1988 ; 3: 347-51$.

5. Wolff RK, Plaetke R, Jeffreys A, White R. Unequal crossing over between homologous chromosomes is not the major mechanism involved in the generation of new alleles at VNTR loci Genomics 1989 ; 5 : 382-4.

6. Armour JAL, Patel I, Thein SL, Fey MF, Jeffreys A. Analysis of somatic mutations at human minisatellite loci in tumors and cell lines. Genomics 1989 ; 4 : 328-34

7. Kelly R, Bulfield G, Collick A, Gibbs M, Jeffreys AJ. Characterization of a highly unstable mouse minisatellite locus : evidence for somatic mutation during early development. Genomics 1989 ; 5 : 844-56.

8. Jeffreys AJ, Neumann R, Wilson V Repeat unit sequence variation in minisatellites : a novel source of DNA polymorphism for studying variation and mutation by single molecule analysis. Cell $1990 ; 60$ : 473-85.

9. Dover GA. DNA fingerprinting. Mapping "frozen accidents". Nature 1990 ; 344 : 812-3. 10. Dover GA. DNA fingerprints. Victims or perpetrators of DNA turnover? Nature 1989 ; 342: 347-8.

Un bulletin trimestriel, Fingerprint News, est édité depuis 1989 par B. Amos et J. Pimberton, Dept of Genetics, Cambridge Univ, Dow ning street Cambrige CB2 3EH, UK. 